DOI: https://dx.doi.org/ 10.33289/IJRPC.10.1.2020.10(4)

\title{
CHARACTERIZATION AND APPLICATION OF DIOLS BASED LIQUID CRYSTALLINE COPOLYESTER
}

\author{
T. Sivaramakrishanan, G. Annamalai and G. Elango* \\ Department of Chemistry, Government Arts College, \\ Tiruvannamalai-606 603, Tamil Nadu, India.
}

\begin{abstract}
Copolyesters are one of the most important classes of polymers with repeating carboxylate group in their spine. A novel random copolyester was synthesized using 1,2-Propanediol, 2,3-Butanediol with phthaloylchloride as dissimilar diols and diacidchloride monomers. The obtained polymers were characterized by Ultraviolet-Visible spectroscopy (UV-Vis), Fourier transform spectroscopy (FTIR), Nuclear magnetic resonance (NMR), Thermogravimetric analysis (TGA) and Differential scanning calorimeter (DSC). The surface morphology and crystalline nature of the polymers were analyzed using Scanning electron microscope (SEM), X-ray diffraction analysis (XRD), and Polarizing optical microscope (POM). Activation energy determination of polyester from their thermal behavior. From the above all details the synthesized polyesters are novel to posses the nature of liquid crystalline. Results of the exploration reveal that the innovative entries of copolymer were recommended into the set of liquid crystalline copolymers.
\end{abstract}

Keywords: Poly(3-oxybutan-2-yl(1-oxypropan-2-yl)Phthalate, Thermal Studies, Activation Energy.

\section{INTRODUCTION}

Polyesters are significant class of high performance and engineering polymers, which find use in a number of diverse applications ${ }^{1-3}$. Polyester is a strong fiber and accordingly can withstand strong and repetitive movements. Its hydrophobic property makes it ideal for garments and jackets that are to be used in wet or damp environments. Coating the fabric with a water-resistant finish intensifies this effect. Since polyester can be molded into almost any shape, certain insulating properties can be built in to the fiber. Polyester is used both as staple fiber as well as continuous filament yarns. Polyester staples are generally used with cotton, wool, and rayon to get blended yarns. Polyester also has industrial uses as well, such as carpet, filters, synthetic artery replacements, ropes, and films. Further, they are also used as film forming, coating, and adhesive, reinforced materials. Different kinds of polyesters have been synthesized over the past decades from various types of diacid chlorides and diols. Thermally stable polyesters derived from isophthalic and terephthalic acids with bisphenolA have been commercialized ${ }^{4}$. A polymeric resin was synthesized by the polycondesation technique of 4-hydroxybenzophenone and melamine with formaldehyde in the presence of $2 \mathrm{M} \mathrm{HCl}$ as a catalyst ${ }^{5}$. However, polyesters are commonly difficult to process because of their limited solubility in organic solvents and their high melting temperature or high glass-transition temperature by virtue of their rigid structures. The polyesters so obtained are in good yield with high molecular weights. Further the range of the polymers prepared is greater. Thus, the growth and expansion of polyester work, both basic and applied over the past few decades has been enormous. In an attempt to synthesize new polyesters by condensation of aliphatic and aromatic diacid chloride with aliphatic diols with liquid crystalline structure, the present work was undertaken ${ }^{6}$.

In this work, we describe the synthesis of novel LCP using 2,3-butane diol using direct polycondensation reaction in the presence of 1,2-Propane diol and phthaloylchloride. Polyesters have found many commercial applications, due to their high crystallinity, they are more difficult to process. 
Surfing of literature implies that less quantum of work has been reported. Hence the scope of present investigation is important as these findings provide a closer insight into the application of polymerization techniques in designing high performance polymers.

\section{MATERIALS AND METHODS}

Chemicals of high purity were obtained from a variety of commercial sources, phthaloylchloride, 2,3butane diol, 1,2-propane diol all purchased from Merk, Mumbai, India. Decontamination of solvent used in this process was 1,2-dichlorobenzene, kept overnight in anhydrous calcium chloride then filtered and purified using fractional distillation method at the temperature of $180^{\circ} \mathrm{C}$ and finally separated. Petroleum ether was dried in presence of anhydrous calcium chloride and finally distilled. Other solvents such as acetone, chloroform, carbontetra chloride, o-chlorophenol, dimethyl sulphoxide (DMSO) and methanol all in AR grade were used to ensure the solubility of the polymer.

\section{Experimental Method}

\section{Synthesis of Poly (3-oxybutan-2-yl(1-oxypropan-2-yl)Phthalate}

The product of the polyester is shown in the Scheme the reaction flask was charged with the 2,3butane diol $(1.8 \mathrm{ml}, 0.02 \mathrm{~mol})$ and propane 1,2 -diol $(0.73 \mathrm{ml}, 0.01 \mathrm{~mol})$. About $150 \mathrm{ml}$ odichlorobenzene was added followed by the phthaloyl chloride $(4.2 \mathrm{ml}, 0.03 \mathrm{~mol})$. The mixture was then refluxed for 65 hours at $160^{\circ} \mathrm{C}$ in nitrogen atmosphere with continuous stirring. The contents were cooled and poured into about $250 \mathrm{ml}$ of petroleum ether. The mixture was kept in refrigerator overnight and filtered. The polyester was dissolved in a minimum amount of acetone and the solution was evaporated to get the pure polyester. It was dried in vacuum over phosphorous pentoxide. The yield was $62 \%$. The formation of the polyester had been confirmed using various spectral techniques viz., UV-Visible, FTIR, ${ }^{1}$ HNMR, TGA and Powder X-rd.

\section{Scheme}

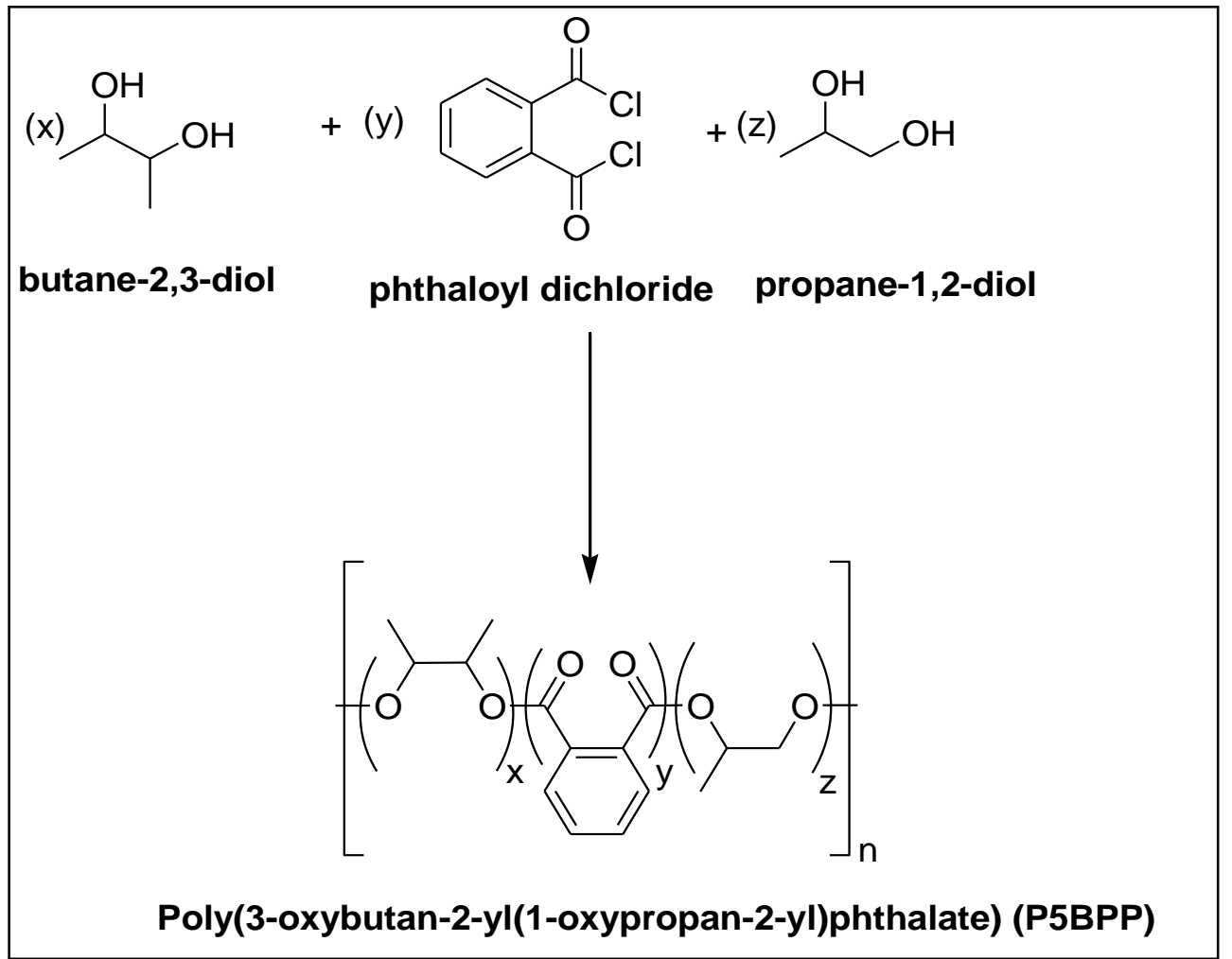

Fig. 1: Synthesis of Polyester

\section{Characterization of Random Copolyester}

The FTIR spectrum of copolyester was reported using Perkin-Elmer spectrophotometer. FTIR Spectrophotometer with the samples incorporated in $\mathrm{KBr}$ pellets. The UV-Visible spectral analysis was performed on Shimadzu-uv-160A spectrophotometer using acetone solution. The ${ }^{1} \mathrm{H}$ NMR spectra were recorded using JEOL GSX 400 FT-NMR spectrometer operating at room temperature. Samples for analysis were prepared by dissolving about $10 \mathrm{mg}$ of the copolyester in $5 \mathrm{ml}$ of spectral grade $\mathrm{CDCl}_{3}$ solvent. Higher magnification pictures were recorded for all co-polyesters with Scanning 
Electron Microscope (SEM).

\section{RESULTS AND DISCUSSION}

\section{Solubility}

Copolyester $10 \mathrm{mg}$ was taken in small stopper test tube which contains $5 \mathrm{ml}$ of the solvent and kept for $24 \mathrm{~h}$ with infrequent shaking. Since, the polyester was insoluble in cold it was slowly heated up to boiling point of the solvent. Thus, the polymer was dissolved. The process was done with different solvent qualitatively.

\section{FTIR Spectrum}

The FTIR Spectrum of Poly(4-oxybutan-2-yl(1-oxypropan-2-yl)phthalate (P5BPP) as shown in Fig- 2. The peak assigned at $1676.65 \mathrm{~cm}^{-1}$ corresponding to carbonyl stretching vibration of the ester group. The absorption peak at $1070.20 \mathrm{~cm}^{-1}$ related to thestretching vibration of the ester $\mathrm{C}-\mathrm{O}$ linkage. The band arising from ortho substituted benzene ring was also present in $2651.84 \mathrm{~cm}^{-1}$. The absorption peak at $736.10,799.35 \mathrm{~cm}^{-1}$ of the aliphatic $\mathrm{C}-\mathrm{H}$ bending vibration ${ }^{7}$. The medium intensity peaks at $2872.34 \mathrm{~cm}^{-1}$ due to aromaticC-H stretching frequency. The peak at $1281.75 \mathrm{~cm}^{-1}$ due to indicating the $\mathrm{CH}_{2}$ wagging present is the synthesized polyester ${ }^{8}$.

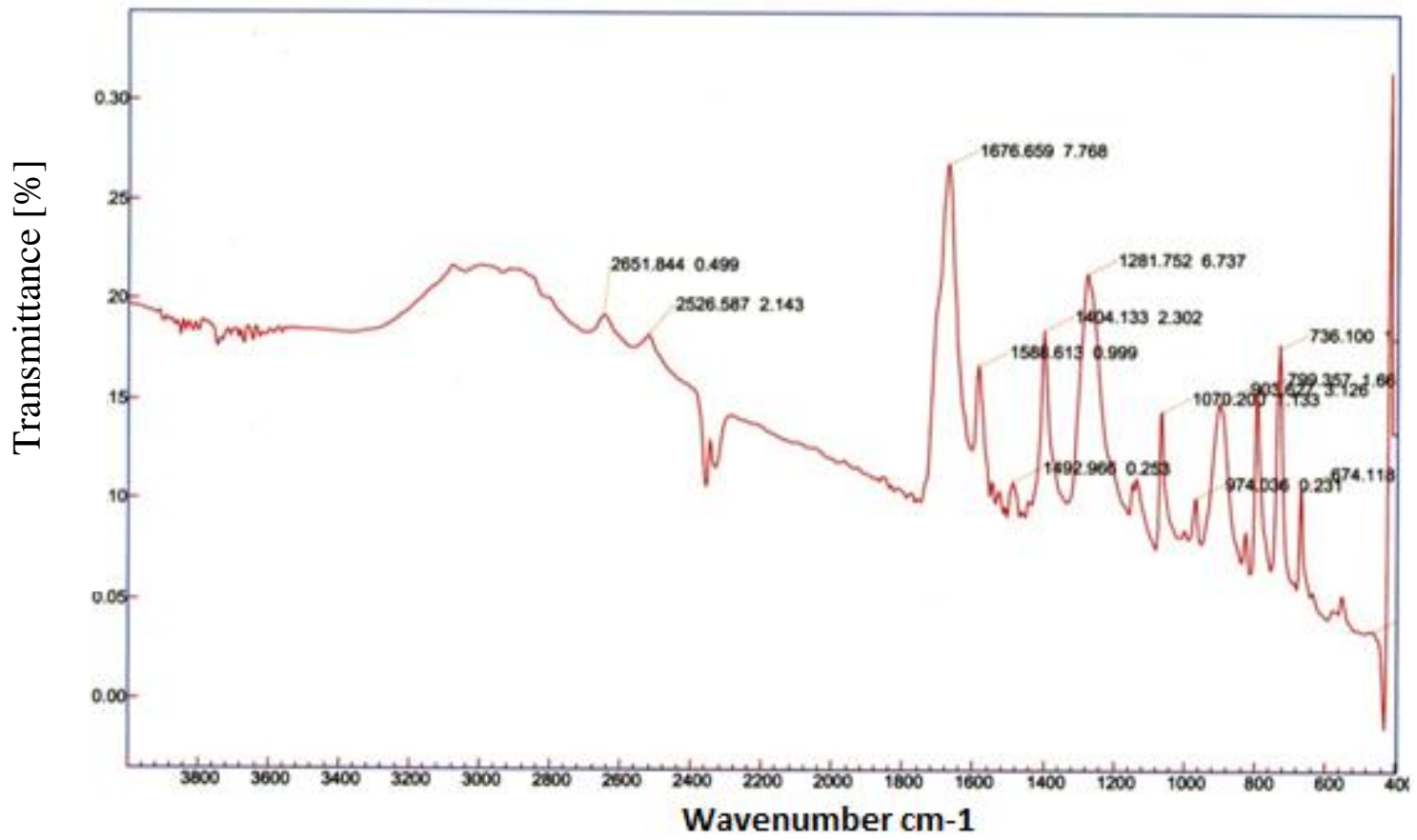

Fig. 2: FTIR Spectrum of Poly(4-oxybutan-2-yl(1-oxypropan-2-yl)phthalate (P5BPP)

\section{${ }^{1} \mathrm{H}$ NMR Spectrum}

${ }^{1} \mathrm{H}$ NMR Spectrum of Poly(4-oxybutan-2-yl(1-oxypropan-2-yl)phthalate (P5BPP) is presented in Fig-3 using $\mathrm{CDCl}_{3}$ as a solvent. The chemical shift values can be explained on the basis of structural units present in these polyesters ${ }^{10}$. A multiplet at $\delta=7.62-7.82 \mathrm{ppm}$ corresponding to aromatic $\mathrm{C}-\mathrm{H}$ protons of the phthaloyl moiety respectively ${ }^{9}$. The two singlet peaks at $\delta=1.16-1.18 \mathrm{ppm}$ were pointed out methyl protons of $2,3-$ butane diol ${ }^{11}$. A singlet at $\delta=1.29 \mathrm{ppm}$ pointed out might be due to methylene protons respectively. 


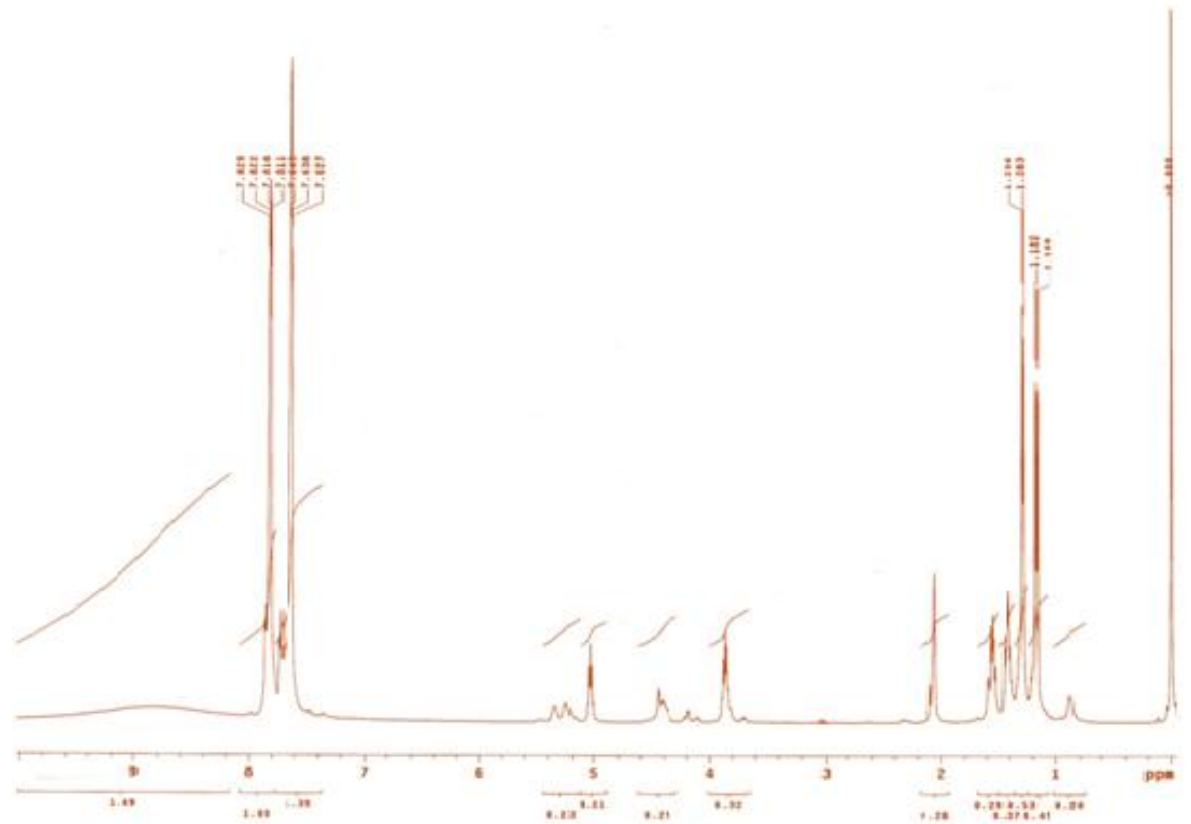

Fig. 3: 'HNMR Spectrum of Poly(4-oxybutan-2-yl(1-oxypropan-2-yl)phthalate (P5BPP)

\section{UV-Visible spectrum}

Poly(4-oxybutan-2-yl(1-oxypropan-2-yl)phthalate (P5BPP) shows two absorption peaks which represented in the Fig-4. The random copolyester reported in the present investigation contains substituted benzene Chromophore. Therefore, there are two possible electronic transitions, namely $\pi$ $\rightarrow \pi^{*}$ and $n \rightarrow \pi^{*}$. The first band arise from $\pi \rightarrow \pi^{*}$ transition with substituted benzene Chromophore at $210 \mathrm{~nm}$. The second band at $230 \mathrm{~nm}$ corresponds to the $\mathrm{n} \rightarrow \pi^{*}$ because it is not influenced by the length of the spacer in the polymer chain, but influenced by the number of condensed rings present in them ${ }^{12}$.

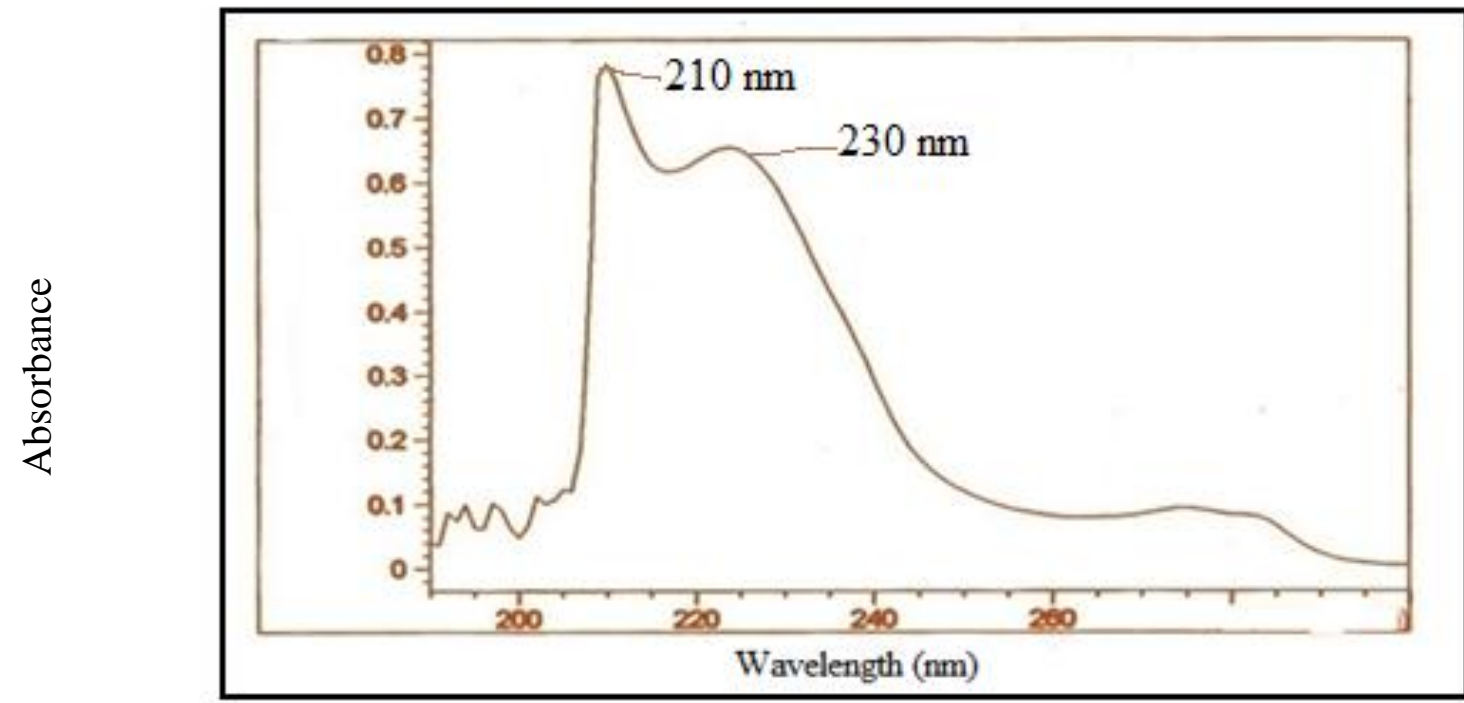

Fig. 4: UV-Visible Spectrum of Poly(4-oxybutan-2-yl(1-oxypropan-2-yl)phthalate (P5BPP)

\section{Thermo Gravimetric Analysis (TGA)}

Fig-5. Thermogram indicates the degradation of Poly(4-oxybutan-2-yl(1-oxypropan-2-yl)phthalate (P5BPP). The degradation of the copolyester was carried out in nitrogen atmosphere at a heating rate of $20^{\circ} \mathrm{C} / \mathrm{min}^{13}$. These thermograms propose that the copolyester is thermally stable upto $175^{\circ} \mathrm{C}$. The first degradation step starts around at $175^{\circ} \mathrm{C}$ and about $10 \%$ of the copolyester was degraded in this step. The second stage of degradation starts around at $275^{\circ} \mathrm{C}$ and in the state approximately $97 \%$ of the copolyester was degraded in this step. 
It is evident from the degradation temperature of copolyesters that the P5BPP polyester degraded at a lower temperature compared to other polymers. This may be due to the presence of aliphatic mesogens ${ }^{14}$. The thermal stability increases with decreasing spacer lengths ${ }^{15}$. It is attributed to the presence of ester linkage in the spacers were expected to introduce significant flexibility and consequently brought down thermal stability ${ }^{16}$.

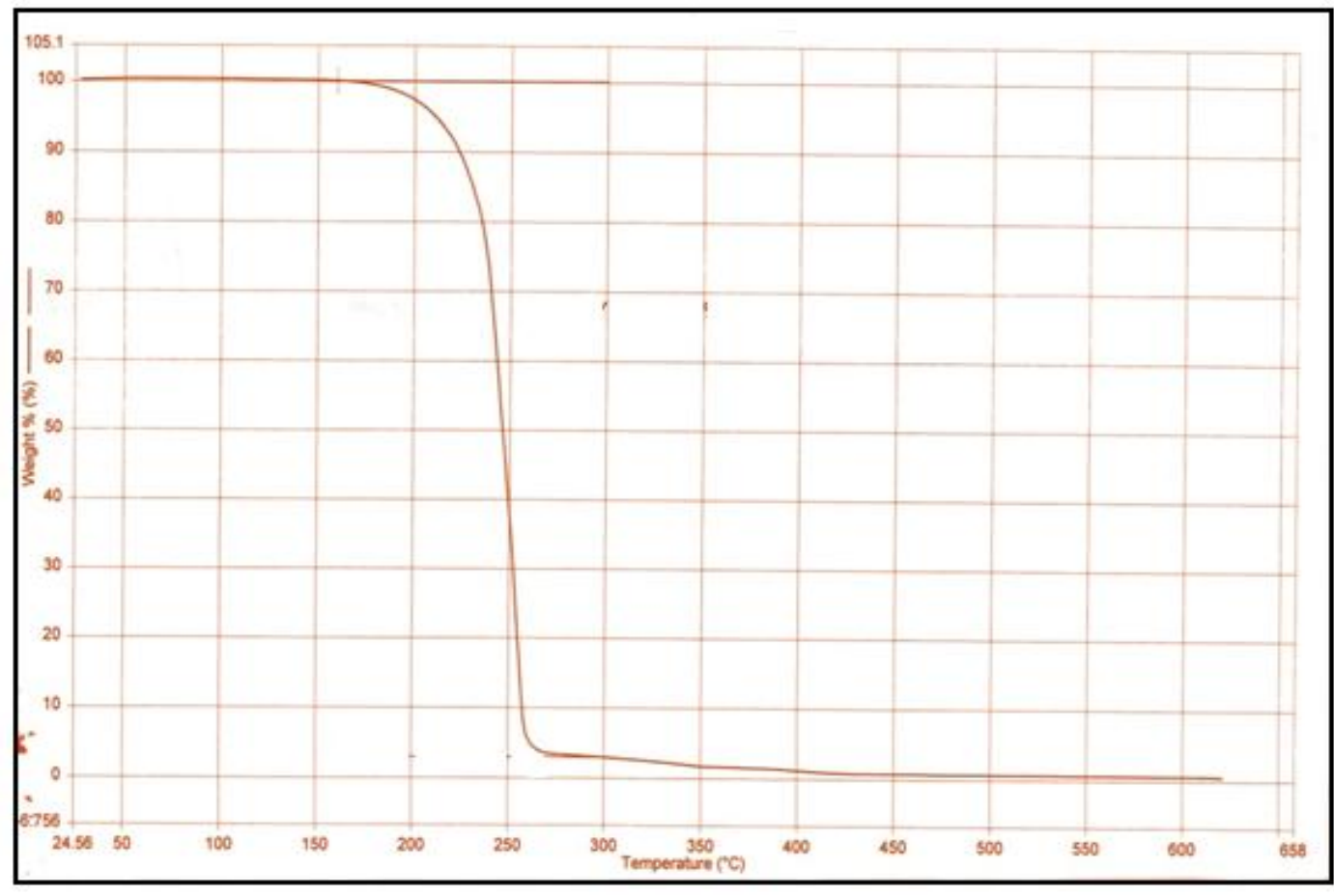

Fig. 5: TGA Thermogram of Poly(4-oxybutan-2-yl(1-oxypropan-2-yl)phthalate (P5BPP)

\section{Differential Scanning Calorimeter (DSC)}

DSC examination was carried out on a TA DSC Q100 calorimeter with a programmed heating procedure in nitrogen atmosphere. The sample size was about $5 \mathrm{mg}$ and encapsulated in hermetically sealed aluminum pans, whose weight were kept constant. The temperature and heat flow scale at different cooling and heating rates were calibrated using standard materials such as indium and benzoic acid ${ }^{17}$.

Thermal analysis of polyesters is important because these investigations not only explain the behavior of the co polyesters when subjected to high temperatures but also aid in establishing uses. In the present investigation, Differential Scanning Calorimetry (DSC) is used to detect the phase transitions in the copolyester P5BPP ${ }^{18}$. DSC thermo grams contain sharp as well as broad peaks at characteristic temperatures indicating phase transitions, before melting. Along with the type of mesophase detected in hot-stage polarizing microscope. These transition temperatures for the formation of mesophase determined by polarizing optical microscope ${ }^{19}$. 


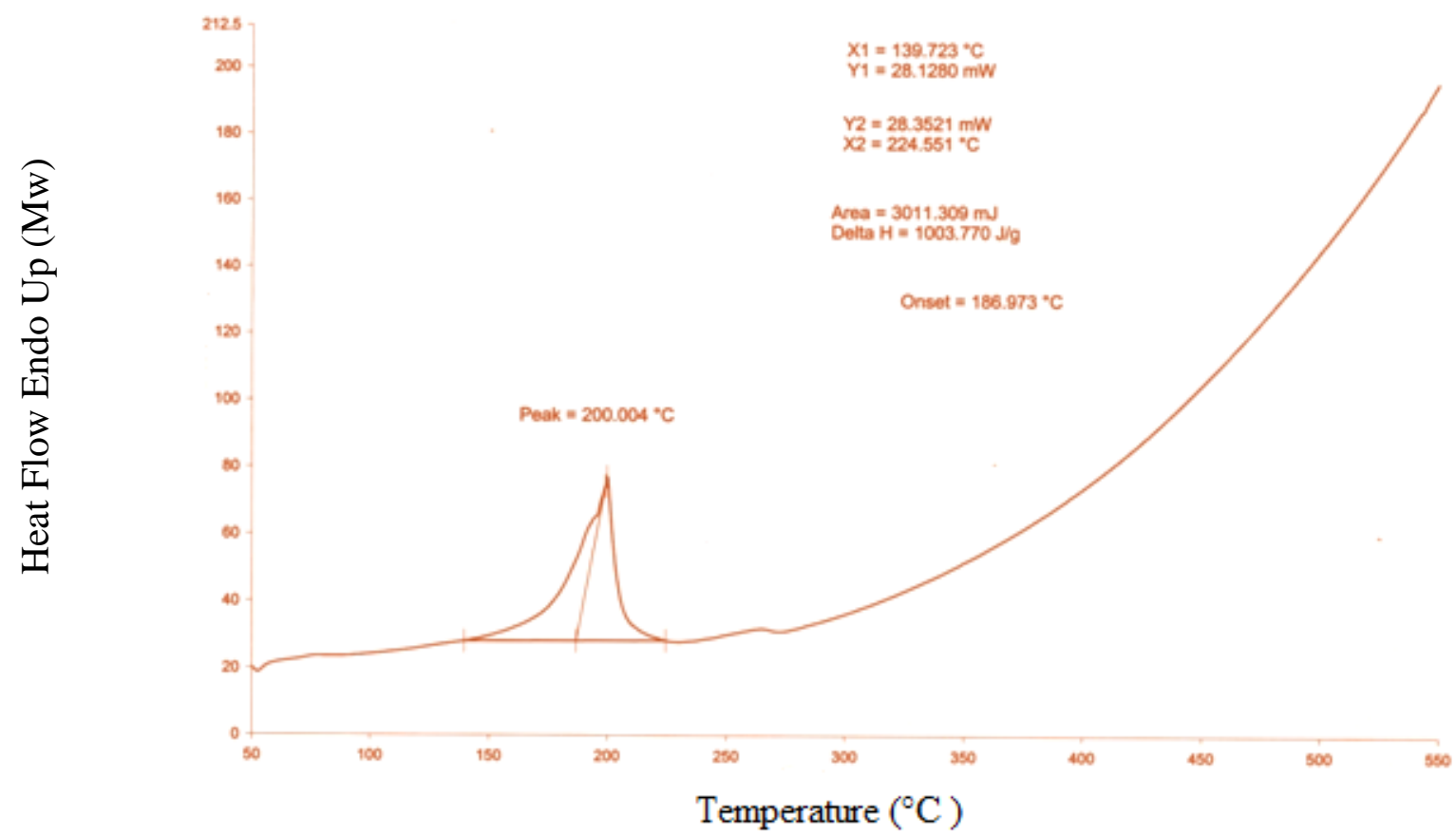

Fig. 6: DSC Thermograms of Poly(4-oxybutan-2-yl(1-oxypropan-2-yl)phthalate (P5BPP)

\section{Hot Stage Optical Polarizing Microscopic Studies}

The characterization of liquid crystals by hot stage optical polarizing microscopy is the most useful for polymers possessing preferred mechanical, thermal and optical properties ${ }^{20}$. The widely employed technique to identify the mesophase in liquid crystalline polyesters is by optical polarising microscopic investigation $^{21}$. Extensive investigation on low molecular mass liquid crystals have proved that LC state can be directly correlated with the chemical constitution of the molecules ${ }^{22}$. Owing to their chemical constitution, liquid crystalline phases having defined molecular organizations are formed. Fig-7 POM image observation present in side chain liquid crystalline polymer, the length of flexible spacer in the side chain determine the mesophase of the polymer P5BPP. If the number of methylene units is less than 6 , nematic mesophase often occurs, Where as smactic mesophase dominates if $n>$ 6. P5BPP have nematic textures. These two consist unsubstituted phenylene rings and aliphatic mesogens. So that they exhibit nematic texture.

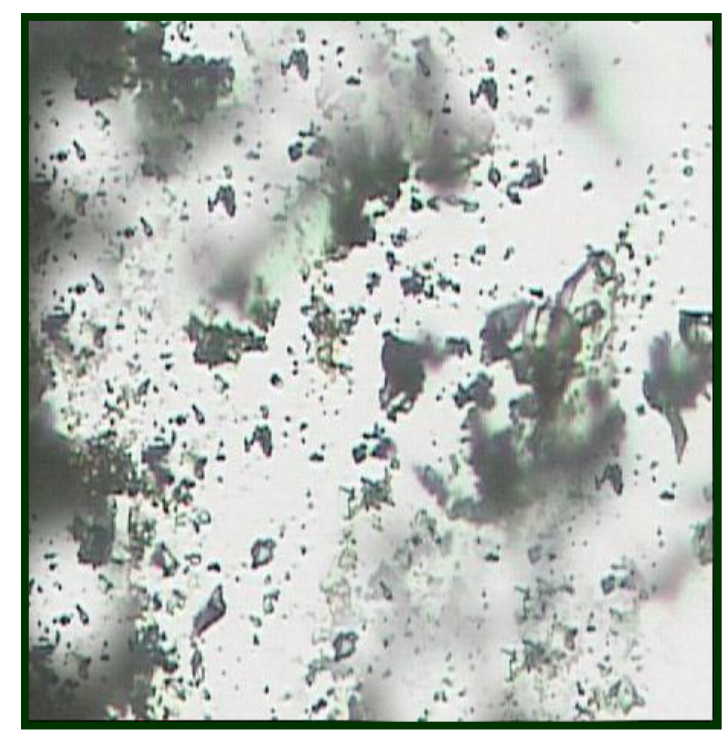

Fig. 7: P5BPP at $220^{\circ} \mathrm{C}(40 \mathrm{X})$ 


\section{Morphology study}

The surface morphology of the Poly(4-oxybutan-2-yl(1-oxypropan-2-yl)phthalate (P5BPP) was studied using SEM image is shown in Fig -8. The rigid and long range orientational order is evident from the SEM images of these polyester P5BPP. It has been suggested that the microstructure of LC polymeric materials have some evidences of crystallinity embedded within extended chain structure of the mesophase ${ }^{23}$.These crystallinity have beendescribed as a non-periodic layer structure which propagates among adjacent oriented chains. The SEM images of these copolyesters show such crystallinity on their film surface indicating long range orientational order $^{24}$.

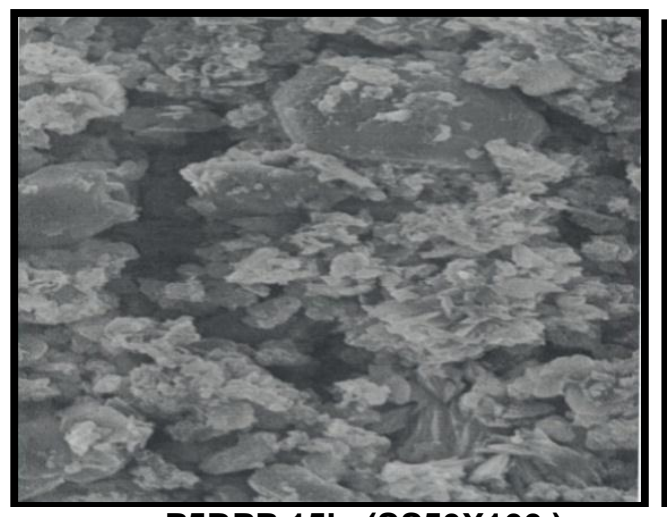

P5BPP 15kv(SS50X100)

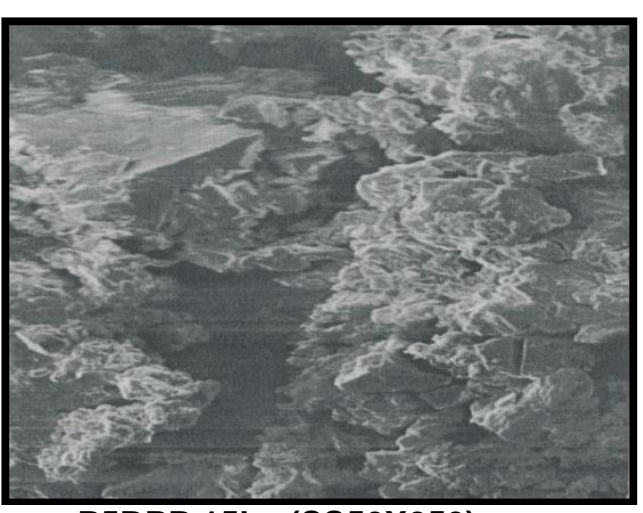

P5BPP 15kv (SS50X250)

Fig. 8: SEM image of Poly(4-oxybutan-2-yl(1-oxypropan-2-yl)phthalate (P5BPP)

\section{X-ray Diffraction Study}

The crystal lattice parameters of the Poly(4-oxybutan-2-yl(1-oxypropan-2-yl)phthalate (P5BPP) was measured by recording X-ray diffractogram given in the Fig-9. The cell parameters data of P5BPP along with the powder diffraction data were listed in Table-1. The polyester P5BPP shows many high intensity peaks. At around $2 \theta=20.5^{\circ}$ the peak intensity is maximum.

This shows that the particular plane (hkl) has higher crystallinity when compared to all other planes. The Full Width Half Maximum (FWHM) of this particular plane is very small, shows the higher crystallinity. This plane can be used as high strength fibres in helmets and bullets. This particular orderly plane is used to develop an increasing variety of new materials with strong and light weight properties. The $2 \theta$ values are from $17-25^{\circ}$ with intermolecular spacing of 4.5 to $4.8 \mathrm{~A}^{\circ}$ have been reported crystalline nature. P5BPP polyester has spacing of about the same order indicating that this polyester exhibits crystalline nature ${ }^{25-26}$.

Table 1: X-RD Diffraction data of the Poly(4-oxybutan-2-yl(1-oxypropan-2-yl)phthalate(P5BPP)

\begin{tabular}{|c|c|c|c|c|}
\hline Pos[2 $\theta$ ] & Height[cts] & FWHM & d-Spacing[A ${ }^{0}$ ] & Rel.int[\%] \\
\hline 20.5 & 97.09 & 0.14 & 4.7 & 100.0 \\
\hline
\end{tabular}




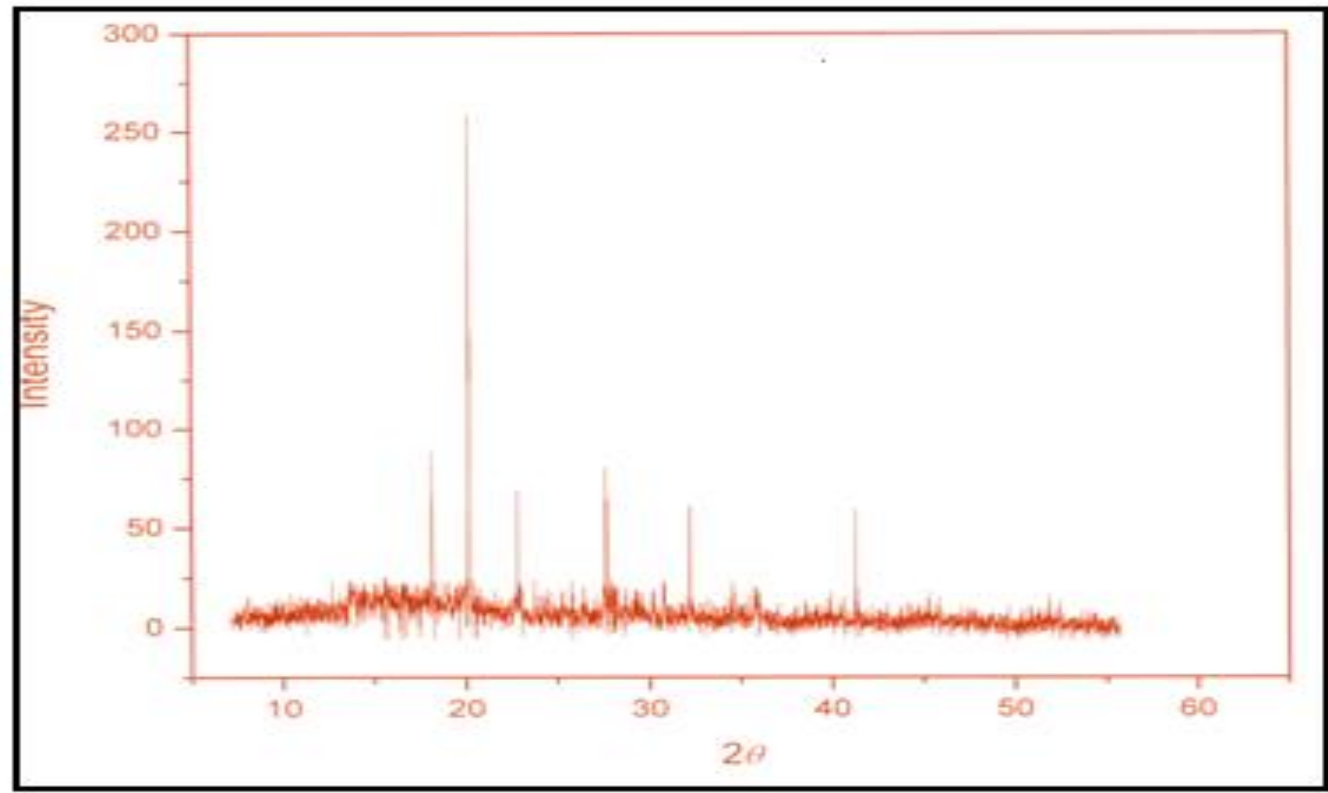

Fig. 9: X-ray Diffractogram of Poly(4-oxybutan-2-yl(1-oxypropan-2-yl)phthalate (P5BPP)

\section{Activation Energy Determination of Polyesters from their thermal Behaviour}

Activation energies (Ea) were calculated graphically using Murray and White, Coats and Redfern and Doyle methods ${ }^{27-29}$. TGA experiments were performed to expose the thermal behaviour and applied the data to calculate the activation energies of polyesters.

The activation energy calculation from TGA curves of the copolyester are given in Table-2

\begin{tabular}{|c|c|c|c|c|c|c|c|c|c|c|}
\hline$\%$ Degradation & $\mathrm{T}(\mathrm{K})$ & $\begin{array}{c}\mathrm{T}^{-1} \times 10^{-3} \\
\mathrm{~K}^{-1}\end{array}$ & $1-C$ & $2 \ln T$ & $\mathrm{~T}^{2}$ & $\ln (I-C)$ & $\begin{array}{c}\log [\ln \\
(1-C)]\end{array}$ & $\begin{array}{c}\ln [\ln (1- \\
\text { C)] }\end{array}$ & $\begin{array}{c}\ln [\ln (I-C)] \\
-2 \ln T\end{array}$ & $\log \left[\ln (1-C) / T^{2}\right]$ \\
\hline 10 & 506.00 & 1.9762 & 90 & 12.453 & 256036 & 4.50 & 0.653 & 1.504 & -10.95 & $2.55 \times 10^{-6}$ \\
\hline 20 & 522.50 & 1.9139 & 80 & 12.517 & 273006 & 4.40 & 0.643 & 1.481 & -11.03 & $2.00 \times 10^{-6}$ \\
\hline 30 & 529.10 & 1.8900 & 70 & 12.542 & 279947 & 4.24 & 0.627 & 1.444 & -11.09 & $1.76 \times 10^{-6}$ \\
\hline 40 & 532.40 & 1.8783 & 60 & 12.555 & 283450 & 4.10 & 0.612 & 1.410 & -11.14 & $1.50 \times 10^{-6}$ \\
\hline 50 & 535.70 & 1.8667 & 50 & 12.567 & 286974 & 3.90 & 0.591 & 1.361 & -11.20 & $1.30 \times 10^{-6}$ \\
\hline 60 & 539.00 & 1.8553 & 40 & 12.579 & 290521 & 3.70 & 0.568 & 1.308 & -11.27 & $1.13 \times 10^{-6}$ \\
\hline 70 & 542.30 & 1.8440 & 30 & 12.592 & 294089 & 3.40 & 0.531 & 1.224 & -11.36 & $6.00 \times 10^{-7}$ \\
\hline 80 & 548.90 & 1.8218 & 20 & 12.616 & 301291 & 3.00 & 0.477 & 1.099 & -11.51 & $9.90 \times 10^{-7}$ \\
\hline 90 & 558.80 & 1.7895 & 10 & 12.652 & 312257 & 2.30 & 0.361 & 0.833 & -11.81 & $8.60 \times 10^{-7}$ \\
\hline
\end{tabular}

Murray and White method

Integral form of Murray and White method can be written as

In this method, plots are plotted in between

$$
\int_{T_{0}}^{T} e^{-E / R T}=\left(R T^{2} / E\right) e^{-E / R T}
$$

$$
\ln [\ln (1-c)]-2 \ln T \text { Vs } T^{-1} \times 10^{-3}\left(K^{-1}\right)
$$




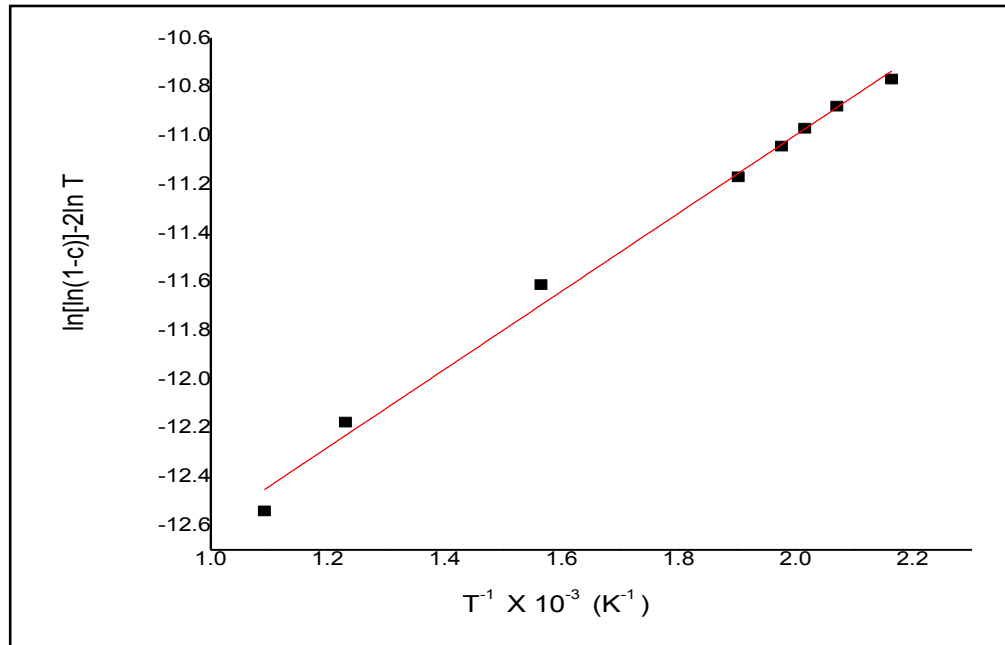

Fig. 10: Arrhenius Plots of Degradation of

Copolyester(Murray and White Method)

\section{Coats and Redfern Method}

Integral form of Coats and Redfern method can be given as

$$
\int_{T_{0}}^{T} e^{-E / R T} d T=\left(R T^{2} / E\right)[1-2 R T / E] e^{-E / R T}
$$

Here plots are plotted against

$$
\log \left[\frac{\ln (1-\mathrm{c})}{\mathrm{T}^{2}}\right] \text { Vs } T^{-1} \times 10^{-3}\left(K^{-1}\right)
$$

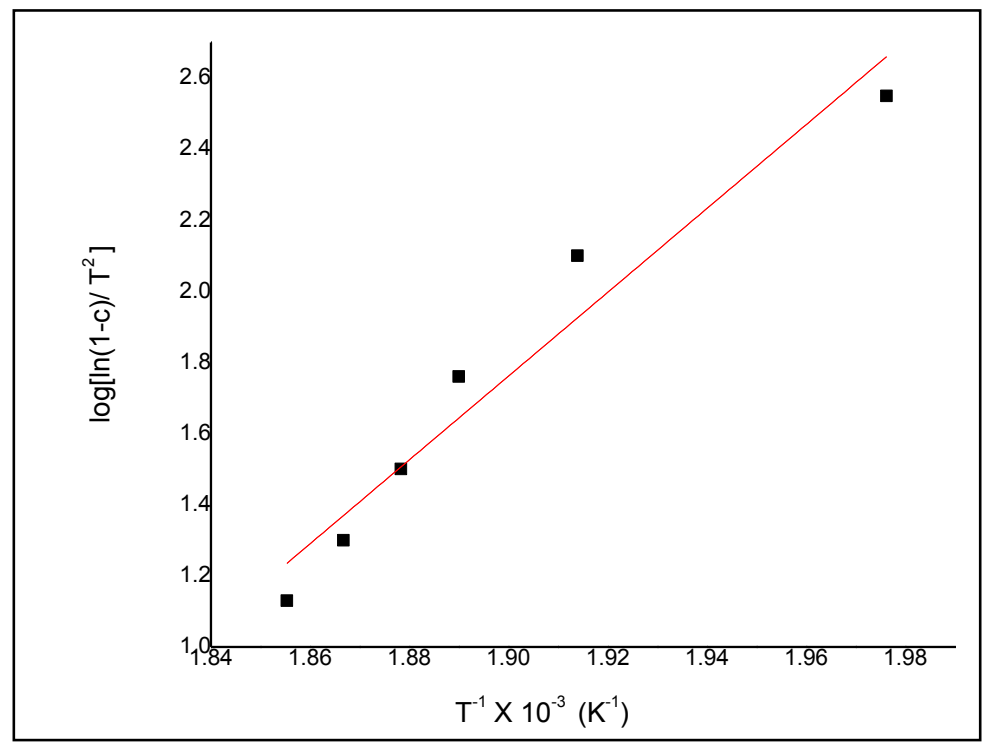

Fig. 11: Arrhenius Plots of Degradation of

Copolyesters (Coats and Redfern Method)

\section{Doyle method}

Integral form Doyle method can be given as

$$
\log \frac{R}{E} \int_{T_{0}}^{T} e^{-E / R T} \cdot d T=-2.315-0.4567(E / R T) \text { for } 20 \geq E / R T \geq 60 \text {. }
$$

In this method the plots are plotted in between

$$
\log [\ln (1-c)] \text { Vs } T^{-1} \times 10^{-3}\left(K^{-1}\right) .
$$




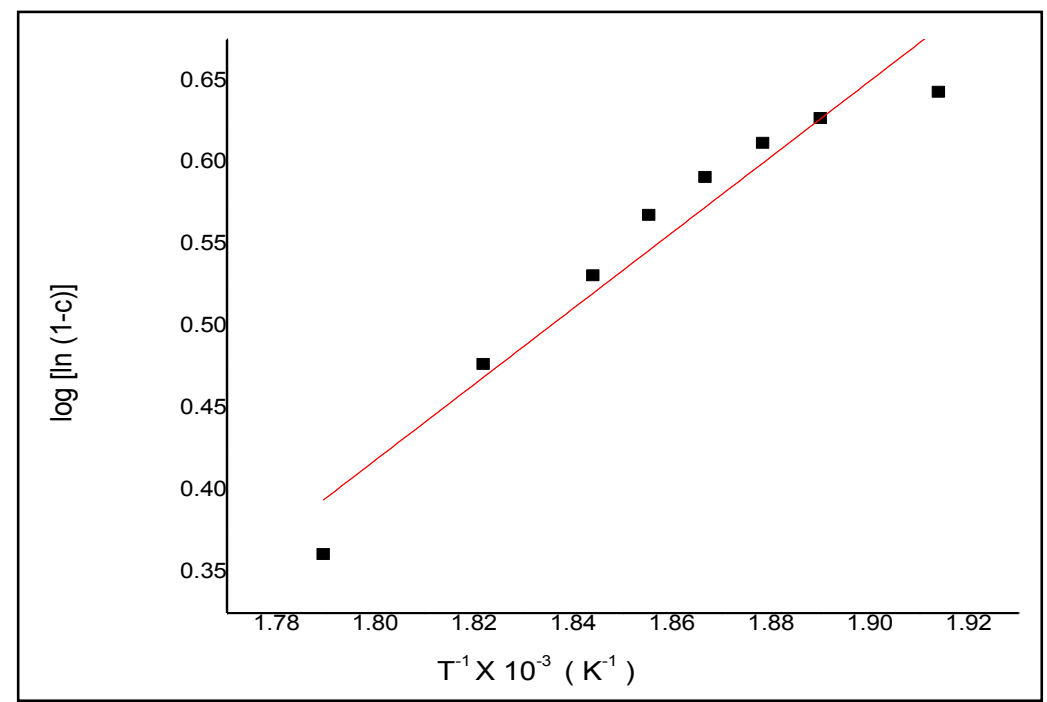

Fig. 12: Arrhenius Plots of Degradation of Copolyesters (Doyle Method)

The activation energies obtained from Murray and White, Coats and Redfern and Doyle method are all in good agreement with each other within the experimental error.

\section{CONCLUSION}

A novel thermally stable liquid crystalline polyester containing 1,2-Propane diol moiety has been synthesized. The reaction pathway involving solution polycondensation technique was used. The copolyester was soluble in common solvents like acetone and chloroform and was further analyzed by different techniques. The chemical structure of the synthesized polyester was confirmed by FTIR and ${ }^{1} \mathrm{H}$ NMR spectral values are in accordance with functional group-ester linkage, protons of the polymer and the nature of mesogens present. The degradation of the polyester was investigated and the thermal analysis reveals the glass transition temperature and melting mesophase formation temperature which are useful in determining the liquid crystalline nature of the polyester, which was further proved by SEM micrograph and XRD pattern providing useful information regarding the crystal of the polymer structure. The molecular weight has a drastic influence on the phase transition temperature of the polyester. Based on the obtained results, the compounds of present investigation may be recommended for future technological and industrial applications.

\section{ACKNOWLEDGEMENTS}

The authors would like to acknowledge Dr. M. Subramani, Principal, and Dr. A. Arun, HOD, PG \& Research Department of Chemistry, Govt. Arts. College, Tiruvannamalai for their support in carrying out this work.

\section{REFERENCES}

1. Seymour RB and Kirshenbaum GS. High performance polymers: their origin and development. NewYork: Elsevier. 1986.

2. Han H and Bhowmik PK. Prog Polym Sci. 1997;22:1431-502.

3. Jadhav JY, Kantor SW and Kroschwitz JI. High performance polymers and composites. New York,Wiley. 1991.

4. Cassidy PA. Thermally stable polymers. New York. Dekker. 1980.

5. Wasudeo B, Gurnule and Sonali P Dhote. Journal of Chemical and Pharmaceutical Research. 2013;5(12): 942-949.

6. Wang Z, Macosko CW and Bates FS. ACS applied materials and interfaces. 2015;8:754-761.

7. Lettieri $M$, Guadagno $L$ and Vittoria V. Effect of physical treatments on the structural organization of a thermotropic polyester, polymer. 1998; 39:2557-2562.

8. Wang Z, Macosko CW and Bates FS. ACS applied materials and interfaces. 2015;8:754-761.

9. Kalsi PS. Spectroscopy of organic compounds, New Age International (P) Ltd., New Delhi, India. 1999.

10. Grobelny $\mathrm{J}$ and Sek D. Investigation of graft copolymer compatibilizers for blends of polyethylene and liquid crystalline polyester and NMR study. Polymer. 1998;39:2143-2147. 
11. Kasmi NZ and Terzopoulou G Z. Papageorgiou Poly(1,4-cyclohexanedimethylene 2,6naphthalate) polyester with high melting point: Effect of different synthesis methods on molecular weight and properties.express Polymer Letters . 2018;12: 227-237.

12. Ebewele RO. Polymer science and technology. CRC press. 2000.

13. Jiang Y, Albert W, Alberda K and Katja L. Biomolecules. 2013; (3):461-480.

14. Motawie MA, Ahmed NM, EIMesallamy SM, Sadek EM and Kandile NG. IOSR Journal of Applied Chemistry. 2014;7: 34-43.

15. Middleton JC and Tipton AJ. Biomaterials. 2001;21: 2335-2346.

16. Indira RT, Tamizharuvi TV, Rajendran and Jaisankar V. Indian Journal of Advances in Chemical Science. 2013;(4): 250-255.

17. Skarja GA and Woodhouse KA. Journal of Applied Polymer Science. 2000;75:1522-1534.

18. Lei L, Ding T, Shi R, Liu Q, Zhang DC and Tian W. Polymer Degradation and Stability. 2007;92: 389-396.

19. Song DK and Sung YK. Journal of Applied Polymer Science. 2003;56:1381-1395.

20. Masatoshi tokita and Kazuya. Ryohei Unusal chain configuration of main- chain liquid crystalline polyester, having Y-shaped mesogens in nematic phase polymer. 2011;52:58305835.

21. Yuanyuan Fang, Yubin Zheng and Fangyuan HU. Synthesis, Characterization and degradation of well-defined crosslinkable aliphatic polyesters end-capped by biomesogenic units, Polymer degradation and stability. 2012;97:185-191.

22. Gray GW. The Molecular Physics of Liquid Crystals, Eds., Luckhurst, G.W. and Gray G.W., Academic Press, New York. 1979.

23. Butzbach GD, Wendorff JH and Zimmermann HJ. Makromol. Chem. Rapid Commun. 1985; 6:821.

24. Zhang W, Jin JII and Lenz RW. Makromol. Chem. 1988;189:2219.

25. Limura K, Koide N and Ohta R. Rep Prog Polym Phys Jap. 1981;24:231.

26. Ober CK, Jin JI and Lenz RW. Makromol. Chem. Rapid Commun. 1983;4:49.

27. Murray $P$ and Whites J. Trans Br Ceram Soc. 1955;54:151.

28. Coats AW and Redfern JP. Nature, London. 1964;201:68.

29. Doyle CD. Journal Applied polymer science. 1962;6:639.

30. Lakshmi L. Activation energy determination of polyimide film from its behavior Asian journal of chemistry. 2010;22(7):5344-5352. 